\title{
Conjugation of Nucleic Acids and Drugs to Gold Nanoparticles
}

\author{
Paula Milán-Rois, Ciro Rodriguez-Diaz, Milagros Castellanos, \\ and Álvaro Somoza
}

\begin{abstract}
Gold nanoparticles (AuNPs) can be used as carriers for biomolecules or drugs in cell culture and animal models. Particularly, AuNPs ease their internalization into the cell and prevent their degradation. In addition, engineered AuNPs can be employed as sensors of a variety of biomarkers, where the electronic and optical properties of the AuNPs are exploited for a convenient, easy, and fast read out. However, in all these applications, a key step requires the conjugation of the different molecules to the nanoparticles. The most common approach exploits the great affinity of sulfur for gold. Herein, we summarize the methods used by our group for the conjugation of different molecules with AuNPs. The procedure is easy and takes around 2 days, where the reagents are slowly added, following an incubation at room temperature to ensure the complete conjugation. Finally, the unbound material is removed by centrifugation.
\end{abstract}

Key words Gold nanoparticles, Spherical nucleic acid, Functionalization, Oligonucleotides, Nanomedicine, Metal nanoparticles, Conjugation, Drug delivery, Sensors

\section{Introduction}

Oligonucleotides and drugs face some challenges for their optimal delivery in cells and animal models. Particularly, oligonucleotides (e.g., antisense, gapmers, and siRNAs) usually present low stability and suffer from reduced cell internalization and selectivity $[1,2]$ and, for these reasons, transfection reagents such as lipofectamine are usually employed to improve delivery. On the other hand, drugs can be too hydrophobic and require solubilizing molecules (e.g., dimethylsulfoxide [DMSO], ethanol). However, these kinds of chemicals present critical restrictions such as cytotoxicity or limited loading. To overcome these drawbacks, delivery systems based on nanoparticles can be employed [3]. There are different types of nanoparticles such as liposomes, micelles, dendrimers, inorganic 
particles, carbon-based nanostructures, viral nanocarriers, polymeric, peptide or metallic nanoparticles, etc. [4-12]. Each vehicle presents different characteristics that can be exploited to address specific challenges related to the delivery of bioactive molecules.

Among the different systems, gold nanoparticles (AuNPs) present excellent properties for the delivery of oligonucleotides because of their low toxicity, cost, and particularly their ease of preparation and functionalization [13]. AuNPs can be synthesized in the laboratory through simple methods, such as the one described by Turkevich [14] and detailed in Subheading 3.1.

The properties of AuNPs can be tuned through their modification with oligonucleotides. When the nanoparticles are densely loaded with oligonucleotides, the resulting nanostructures are known as spherical nucleic acids (SNA) [15]. This kind of nanostructure presents interesting features, such as high internalization in a wide variety of cells and low toxicity. Therefore, these derivatives can be employed for multiple applications, such as drug delivery systems, gene therapy and regulation, or molecular diagnosis $[16,17]$.

Regarding the vehiculization of therapeutics, AuNPs can be used for the delivery of hydrophobic drugs such as paclitaxel, doxorubicin, or AZD8055 without affecting their effectiveness $[18,19]$. On the other hand, AuNPs functionalized with oligonucleotides (e.g., siRNAs, gapmers) could be used as a substitute for transfection reagents in different applications involving gene regulation, or even immunomodulatory processes, for the treatment of diseases such as cancer, sepsis, skin disorders, diabetes, etc. [16, 2022].

In the case of diagnostics, it is worth mentioning that fast and accurate point-of-care diagnostic systems are critical in personalized medicine. In particular, nucleic acid detection is of great importance for the diagnosis and treatment of many diseases caused by genetic mutations, infectious agents, or other physiologically abnormal circumstances. Conventional methods such as RT-PCR offer high accuracy and sensitivity; however, these methods are not suitable for routine diagnosis because they are time-consuming and need highly trained personnel and expensive equipment. One development that seems to simplify the nucleic acid detection and we study in the lab is the use of SNA based on a single-stranded oligonucleotide with a unique stem-loop structure (Molecular Beacon, MB) $[23,24]$.

This chapter describes how to conjugate drugs or oligonucleotides to AuNPs, which can be further used as delivery systems of therapeutics and sensors.

To attach any compound to AuNPs, the high affinity of thiol groups to gold could be exploited. Thus, the molecules (e.g., oligonucleotides, drugs) should be functionalized with linkers 
containing sulfur-based moieties, such as thiols or dithiolanes [25], which are commented in this chapter.

AuNPs conjugation requires a few simple steps of addition, incubation, and washes. The method might change slightly depending on the linker employed for the conjugation, which can be designed to control the release or stability of the cargo. In general, the use of dithiolane provides more robust structures and can be achieved in few hours, whereas the use of thiols implies more than 1 day. For the reader's convenience, we have included the preparation of the dithiolane linkers used in our group. The approach can be used for the conjugation of drugs, polymers (e.g., polyethylene glycol [PEG]), or the preparation of oligonucleotides in a DNA synthesizer using a tailored solid support, usually based on controlled pore glass (CPG).

\section{Materials}

\subsection{AuNP Synthesis}

2.1.1 Materials

2.1.2 Reagents
- $250 \mathrm{~mL}$ round-bottom glass flask.

- Septum for a $250 \mathrm{~mL}$ round-bottom flask.

- $3.5 \mathrm{~cm}$ long magnet.

- $0.3 \mu \mathrm{m}$ fritted filter for vacuum filtration.

- $250 \mathrm{~mL}$ Erlenmeyer flask with an output for vacuum.

- Plastic material such as conical centrifuge tubes and microcentrifuge tubes.

- $1 \mathrm{~mL}$ quartz cuvette.

- Gold solution: 945.2 $\mu \mathrm{M}$ Hydrogen tetrachloroaurate (III) hydrate $\left(\mathrm{AuCl}_{4} \mathrm{H}_{3} \mathrm{O}\right)$ in $100 \mathrm{~mL}$ autoclaved Milli- $\mathrm{Q}$ grade water.

- Ultrapure reagent-grade water.

- Sodium citrate solution: $40 \mathrm{mM}$ sodium citrate tribasic dihydrate $(118 \mathrm{mg})$ in $10 \mathrm{~mL}$ autoclaved Milli-Q grade water.

- Hot plate $(7 \mathrm{~cm}$ radius) with magnetic stirring.

- Reflux column.

- Vacuum pump.

- UV-Vis spectrophotometer. 


\subsection{Dithiolane-Based Linkers Synthesis}

\subsubsection{Materials}

2.2.2 Reagents
- $50 \mathrm{~mL}$ round-bottom flask.

- 2 -cm long magnet.

- 1 septum.

- Thin-layer chromatography (TLC) (sheets of silica gel 60F254).

- Filter paper.

- $2000 \mathrm{KDa}$ tubing membrane.

- Lipoic acid.

- N-hydroxysuccinimide (NHS).

- Tetrahydrofuran (THF).

- N, $N^{\prime}$-dicyclohexylcarbodiimide (DCC).

- Ethyl acetate (AcOEt).

- Methoxypolyethylene glycol amine $\left(\mathrm{PEG}-\mathrm{NH}_{2}\right)$.

- Drug with a primary amine (e.g., Gemcitabine).

- Dimethylformamide (DMF).

- Dichloromethane $\left(\mathrm{CH}_{2} \mathrm{Cl}_{2}\right)$.

- Methanol (MeOH).

- Threoninol.

- 4,4'-Dimethoxytrityl chloride (DMTrCl).

- Dry pyridine (Py).

- Hexane.

- Succinic anhydride.

- Dry $\mathrm{CH}_{2} \mathrm{Cl}_{2}$.

- 4-(Dimethylamino)pyridine (DMAP).

- N,N-Diisopropylethylamine (DIPEA).

- Distilled water.

- Magnesium sulfate anhydrous $\left(\mathrm{MgSO}_{4}\right)$.

- 1-Hydroxybenzotriazole $\left(\mathrm{HBO}^{t}\right)$.

- Acetonitrile (MeCN).

- CPG: Aminopropyl-CPG, $1000 \AA$.

- Caping reagent $\mathrm{A}$ (CAP A): THF/pyridine/acetic anhydride (8: $1: 1)$.

- Caping reagent B (CAP B): 10\% Methylimidazole in THF.

- Flash column chromatography using silica gel $(60 \AA$, $230 \times 400$ mesh).

- Rotavapor. 


\subsection{Functionalization of AuNPs}

\subsubsection{Materials}

2.3.2 Reagents

2.3.3 Equipment
- Plastic material: one microcentrifuge tube per condition.

- A 96-well plate for absorbance measurements in a plate reader.

- Oligonucleotides with sulfide-based modifications at micromolar concentration.

- Annealing buffer 3×: $30 \mathrm{mM}$ Tris-HCl, $3 \mathrm{mM}$ EDTA, $150 \mathrm{mM} \mathrm{NaCl}$.

- Tris (2-carboxyethyl) phosphine hydrochloride solution (TCEP).

- Gold nanoparticles (AuNP) with a diameter of $13 \pm 2 \mathrm{~nm}$ (see Subheading 3.1).

- Sodium chloride solution: $5 \mathrm{M} \mathrm{NaCl}$.

- Oligonucleotide quantification kit (e.g., Quant-iT ${ }^{\mathrm{TM}}$ OliGreen $^{\mathrm{TM}}$ ssDNA Assay Kit, Qubit ${ }^{\mathrm{TM}}$ ssDNA Assay Kit), including the Quant-iT OliGreen ${ }^{\circledR}$ ss DNA Reagent, TE $20 \times$ buffer, and oligonucleotide standard.

- Autoclaved Milli-Q grade water.

- PEG modified with a dithiolane group (see Subheading 3.2.2).

- Chemotherapeutic drugs with dithiolane-based linker (e.g., gemcitabine) (see Subheading 3.2.3).

- Benchtop centrifuge.

- Vortex mixer.

- Orbital shaker.

- Plate reader suitable for absorbance and fluorescence determinations using 96-well plates.

- Evaporating centrifuge.

\section{Methods}

3.1 AuNP Synthesis For the preparation of AuNP $(13 \pm 2 \mathrm{~nm})$ the Turkevich method is used [14] as follows:

1. Turn on the heating plate to $140{ }^{\circ} \mathrm{C}$.

2. Add $100 \mu \mathrm{L}$ of $\mathrm{HAuCl}_{4}$ solution $(94.52 \mu \mathrm{mol})$ in a $250 \mathrm{~mL}$ round bottom flask containing $100 \mathrm{~mL}$ of sterile water.

3. Add the magnet to the round bottom flask.

4. Place the round bottom flask in the heating plate while stirring at $700 \mathrm{rpm}$ approximately, with a reflux system, and heat it to reflux. 
3.2 Dithiolane-Based Linkers Synthesis

3.2.1 Compound 1 [2,5Dioxopyrrolidin-1-y/(R)-5(1,2-Dithiolan-3-yl)

Pentanoate]
5. Prepare the sodium citrate tribasic dihydrate solution in a $50 \mathrm{~mL}$ conical centrifuge tube.

6. When the mixture boils, add the citrate solution quickly while stirring at $700 \mathrm{rpm}$.

7. Wait for $15 \mathrm{~min}$ and then remove the round bottom flask from the heating plate. During this period, the color of the solution turns from yellow to red (see Note $\mathbf{1}$ ).

8. Leave the mixture stirring at $300 \mathrm{rpm}$ at room temperature and protect from the light for $16 \mathrm{~h}$.

9. Filter the solution using a $0.3 \mu \mathrm{m}$ fritted filter with the help of vacuum.

10. Determine nanoparticles' size: for proper characterization of gold nanoparticles, the size should be measured by TEM and the concentration determined by UV-Vis spectrophotometry using the Beer-Lambert law. It requires measuring the UV-Vis absorbance at $520 \mathrm{~nm}$ and using the corresponding extinction coefficient $(\varepsilon)$ for its size [26]. For instance, to determine the concentration of $13 \pm 1 \mathrm{~nm}$ AuNPs, which have an extinction coefficient $(\varepsilon)$ of $2.7 \times 10^{8} \mathrm{M}^{-1} \mathrm{~cm}^{-1}$, you should use the following equation (Eq. 1):

$$
\text { concentration }=\frac{A}{\varepsilon \times l}
$$

where $A$ is the absorbance at $520 \mathrm{~nm}, l$ is the optical path in $\mathrm{cm}$, and $\varepsilon$ extinction coefficient in $\mathrm{M}^{-11} \mathrm{~cm}^{-1}$.

The preparation of the dithiolane-based derivatives of drugs (3) and PEG $(2)$ is summarized in Fig. 1 and described in the following instructions. In the case of oligonucleotides, the required solid support $(\mathrm{CPG})$ containing a dithiolane moiety for the preparation of oligonucleotides is also described (7).

1. Dissolve lipoic acid ( 1 equiv.) and $N$-hydroxysuccinimide (1.2 equiv.) in tetrahydrofuran $(0.5 \mathrm{M})$.

2. Stir the solution at $0{ }^{\circ} \mathrm{C}$ for $10 \mathrm{~min}$.

3. Dissolve $N, N^{\prime}$-dicyclohexylcarbodiimide (1.2 equiv.) in tetrahydrofuran $(3.5 \mathrm{M})$ and add it slowly to the lipoic acid and $N$ hydroxysuccinimide solution obtained in step $\mathbf{l}$.

4. Stir the reaction at room temperature for $5 \mathrm{~h}$.

5. Filter the mixture using a filter paper and wash the solid with cold ethyl acetate. Evaporate the solvent under vacuum to obtain compound $\mathbf{l}$ as a yellow oil. 


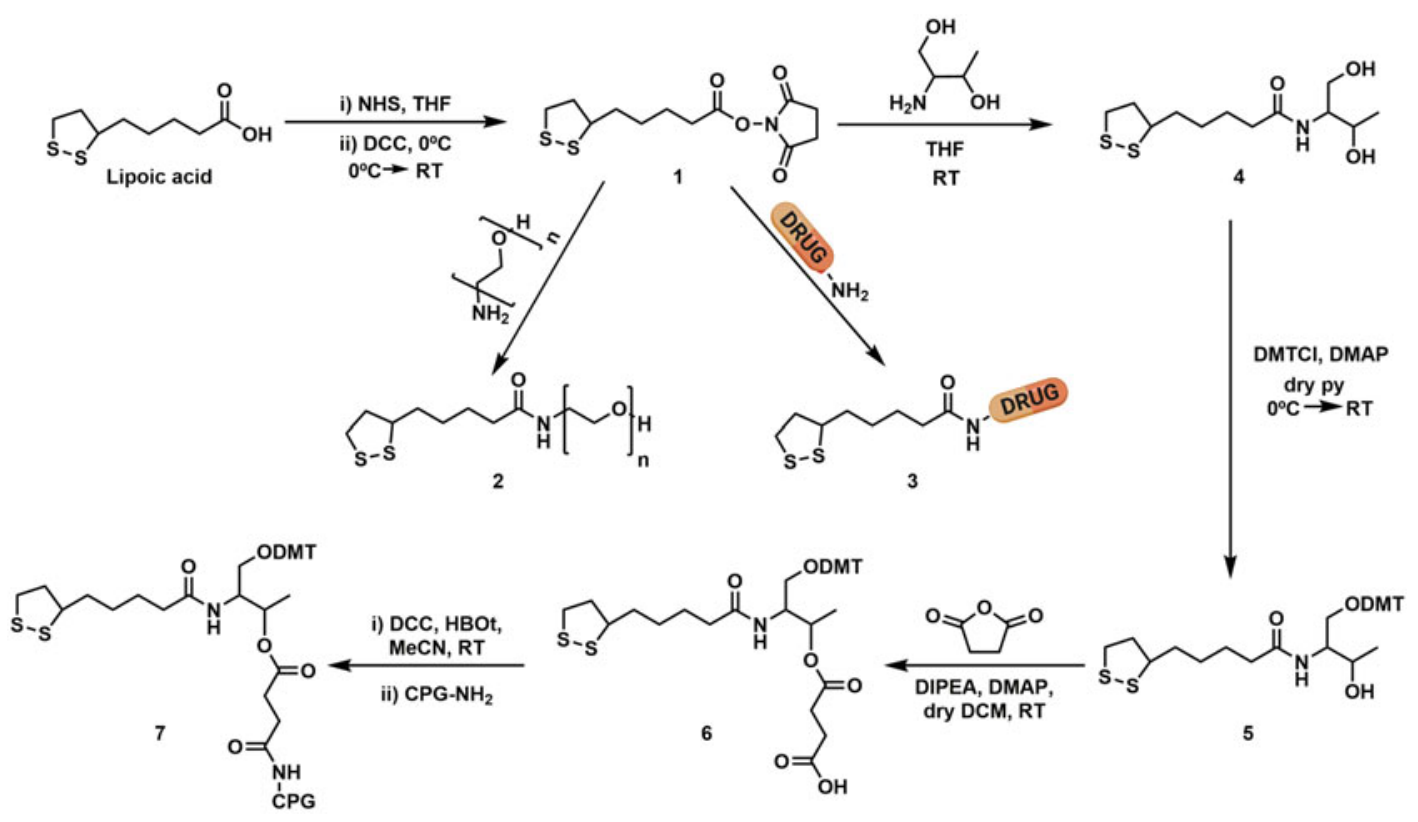

Fig. 1 Schematic representation of the synthesis of dithiolane-modified products: PEG, drug, and CPG

3.2.2 PEG (2)

3.2.3 Drug-Modified Linker (3)
1. Dissolve compound 1 (2 equiv.) and PEG- $\mathrm{NH}_{2}$ (1 equiv.) in tetrahydrofuran.

2. Stir the reaction at room temperature for $18 \mathrm{~h}$.

3. Purify the crude by dialysis with a 2000 KDa tubing membrane.

4. Stir the solution for 18 h to obtain compound 2 .

1. Dissolve compound 1 ( 2 equiv.) and a drug containing a primary amine (e.g., Gemcitabine) (1 equiv.) in dimethylformamide $(0.1 \mathrm{M})$.

2. Stir the reaction at room temperature for $24 \mathrm{~h}$.

3. Eliminate the solvent in vacuum.

4. Purify the crude by flash chromatography $\left(\mathrm{CH}_{2} \mathrm{Cl}_{2}: \mathrm{MeOH} /\right.$ 25:1) to obtain compound 3 .

1. Dissolve compound 1 ( 1 equiv.) and threoninol ( 1.1 equiv.) in $\operatorname{THF}(0.15 \mathrm{M})$.

2. Stir the solution at room temperature for $18 \mathrm{~h}$.

3 . Eliminate the solvent in vacuum.

4. Purify the crude by flash chromatography $\left(\mathrm{CH}_{2} \mathrm{Cl}_{2}: \mathrm{MeOH} /\right.$ 25:1) to obtain compound 4 as a yellow oil.
3.2.4 Compound $4: \mathrm{N}-$

(1,3-Dihydroxybutan-2-yl)-

5-(1,2-Dithiolan-3-yl)

Pentanamide 
3.2.5 Compound 5: N-(1(bis(4-Methoxyphenyl) (Phenyl)Methoxy)-3Hydroxybutan-2-yl)-5-(1,2Dithiolan-3-yl)

Pentanamide
3.2.6 Compound 6: 4((3-)(5-(1,2-Dithiolan-3-yl) Pentanamido)-4-(bis(4Methoxyphenyl)(Phenyl) Methoxy)Butan-2-y()oxy)4-Oxobutanoic Acid

\subsubsection{Compound 7: 4-} ((3-(5-(1,2-Dithiolan-3-yl) Pentanamido)-4-(bis(4Methoxyphenyl)(Phenyl) Methoxy)Butan-2-yl)oxy)4-Oxobutanamide CPG

\subsection{AuNP Functionalization with Thiol-Modified Oligonucleotides}

1. Dissolve compound 4 ( 1 equiv.) in dry pyridine $(0.3 \mathrm{M})$.

2. Dissolve $\mathrm{DMTrCl}$ ( 1.2 equiv.) in dry pyridine ( $1 \mathrm{M})$ and add to the compound 4 solution in dry pyridine at $0{ }^{\circ} \mathrm{C}$.

3. Stir the reaction at $0{ }^{\circ} \mathrm{C}$ for $30 \mathrm{~min}$, then at room temperature for $18 \mathrm{~h}$.

4. Eliminate the solvent in vacuum.

5. Purify the crude by flash chromatography (Hexane:AcOEt/1: 1) to obtain compound $\mathbf{5}$ as a beige foam.

1. Dissolve compound 5 (1 equiv.), DMAP (0.1 equiv.) and DIPEA (1.4 equiv.) in dry $\mathrm{CH}_{2} \mathrm{Cl}_{2}(0.13 \mathrm{M})$.

2. Dissolve succinic anhydride (1.3 equiv.) in dry $\mathrm{CH}_{2} \mathrm{Cl}_{2}(0.3 \mathrm{M})$ and add the solution slowly to the mixture prepared in the previous step at $0{ }^{\circ} \mathrm{C}$.

3. Stir the reaction at room temperature for $18 \mathrm{~h}$.

4. Wash the solution with water 3 times.

5. Dry the organic layer with $\mathrm{MgSO}_{4}$.

6. Eliminate the solvent in vacuum to obtain compound $\mathbf{6}$.

1. Dissolve compound 6 ( 1 equiv.) in $\operatorname{MeCN}(0.05 \mathrm{M})$.

2. Dissolve DCC ( 1 equiv.) and $\mathrm{HBO}^{t}$ ( 1 equiv.) in $\mathrm{MeCN}$ $(0.2 \mathrm{M})$ and add to compound 6 solution at room temperature for $3 \mathrm{~h}$.

3. Filter the solution with filter paper and add to the CPG ( 5 equiv. in $\mathrm{mg}$ ).

4. Stir the mixture for $3 \mathrm{~h}$ at room temperature.

5. Remove the solvent and wash the CPG with $\mathrm{MeOH}$ three times and with $\mathrm{CH}_{2} \mathrm{Cl}_{2}$ three times.

6. Dry the CPG.

7. Add a mixture of capping reagents [CAP A:CAP B $(1: 1)](1 \mathrm{~mL}$ per $175 \mathrm{mg}$ of $\mathrm{CPG})$.

8. Stir the solution for $1 \mathrm{~h}$ at RT.

9. Remove the solvent and wash the CPG with $\mathrm{MeOH}$ three times and with $\mathrm{MeCN}$ three times.

Oligonucleotides can be easily attached to AuNPs using a thiolbased linker, which is commercially available, and most oligonucleotide providers offer this modification. However, the thiol group should be deprotected, as detailed below, before incubating the oligonucleotides with AuNPs (Fig. 2).

1. Incubate the oligonucleotide with TCEP ( see Note 2) using a $100 \times$ excess relative to the oligonucleotide's thiol (see Note 3 ) 
A

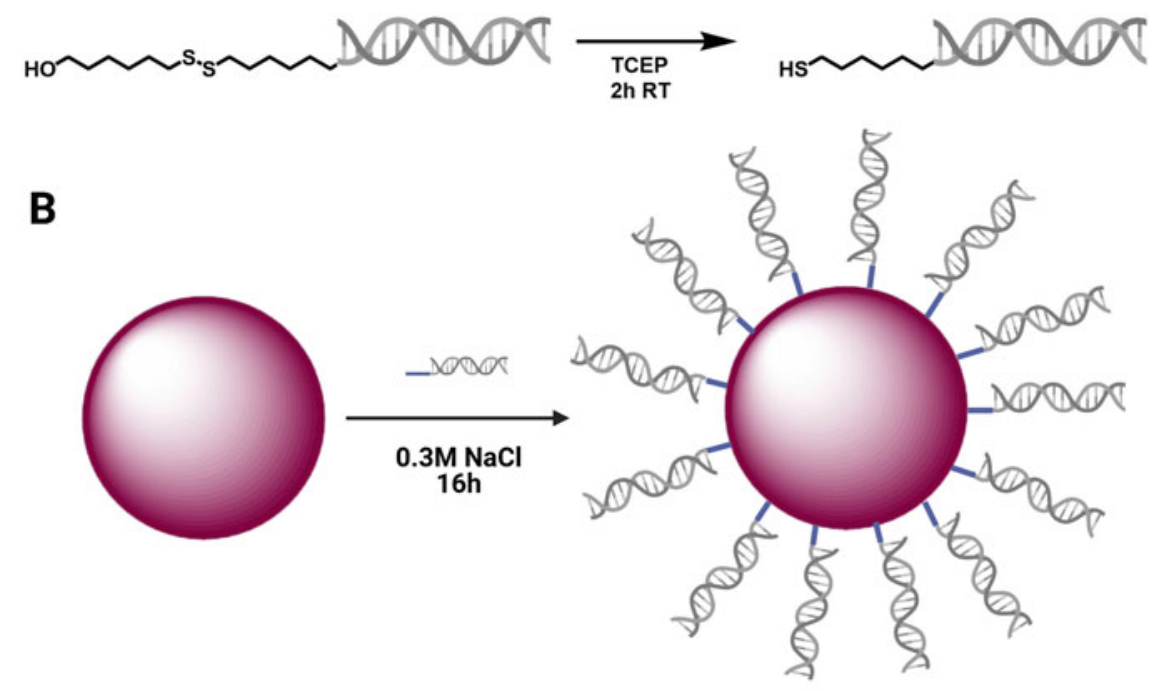

Thiol-modified oligonucleotide -Do00 AuNP

Fig. 2 Schematic representation of: (a) Deprotection of oligonucleotides bearing a thiol moiety. (b) Functionalization of AuNPs with thiol-modified oligonucleotides

for $2 \mathrm{~h}$ at room temperature and moderate agitation on a minishaker (e.g., for deprotect $500 \mu \mathrm{L}$ of an oligonucleotide solution at $20 \mu \mathrm{M}$ (i.e., $20 \mathrm{pmol} / \mu \mathrm{L}$ ) use $2 \mu \mathrm{L}$ of TCEP at $0.5 \mathrm{M}$ ) (see Note 4).

2. Add the deprotected oligonucleotide slowly to the AuNP solution prepared at $12 \mathrm{nM}$.

3 . Incubate the mixture for $45 \mathrm{~min}$ at room temperature and moderate agitation.

4. Add $60 \mu \mathrm{L} \mathrm{NaCl}$ solution to a final concentration of $0.3 \mathrm{M}$ through the addition of small volumes (e.g., 5-10 $\mu \mathrm{L})($ see Note 5). Vortex the solution quickly after each addition of $\mathrm{NaCl}$ solution and incubate the sample for at least $10 \mathrm{~min}$ on a mini-shaker between each addition.

5 . Incubate the sample for $16 \mathrm{~h}$ at room temperature on a minishaker with moderate agitation.

6. Remove any unbound material by centrifugation at $13.2 \mathrm{rpm}$ and $4{ }^{\circ} \mathrm{C}$ for $30 \mathrm{~min}$. After the centrifugation, remove the supernatant and save it for later use. The pellet should be resuspended by vortexing in sterile water using the same volume of water removed to keep the concentration constant. Repeat the cleaning step 3 times ( see Note 6).

7. Evaporate the collected supernatants and use the Quant-iTTM OliGreen $^{\mathrm{TM}}$ ssDNA Assay Kit protocol to determine the 


\subsection{Oligonucleotide Quantification}

3.4.1 Standard Curve Preparation

3.4.2 Oligonucleotide Quantification in AuNPS

\subsection{AuNP}

Functionalization with

Dithiolane-Modified Oligonucleotides or Drugs

3.5.1 AuNP

Functionalization with Dithiolane-Modified Oligonucleotides unbound oligonucleotide from the solution (see Subheading 3.4) (see Note 7).

For the quantification of oligonucleotides in the supernatant, use an oligonucleotide quantification kit. In this case, the Quant-iT OliGreen kit is used. The general procedure is as follows.

1. Prepare a standard curve for each oligonucleotide using at least 5 dilutions of the specific oligonucleotide in TE buffer $(1 \times)$, e.g., $0,1000,750,500,250 \mathrm{ng} / \mathrm{mL}$.

2. Prepare a solution of Quant-iT OliGreen ${ }^{\circledR}$ ss DNA Reagent $(2 \mu \mathrm{g} / \mathrm{mL})$ in TE $(1 \times)$.

3. Mix each oligonucleotide dilution from step 1 with $1 \mathrm{~mL}$ Oligreen solution prepared in step 2. Incubate the solution for $5 \mathrm{~min}$ at room temperature protected from light.

4. Take $200 \mu \mathrm{L}$ of each solution prepared in step 3 and measure it in a plate reader (excitation $480 \mathrm{~nm}$, emission $520 \mathrm{~nm}$ ).

5. Plot the data in a concentration $(\mathrm{ng} / \mathrm{mL}, X$ axis) vs. absorbance (a.u., $\Upsilon$-axis) graph. Fit the data to a simple linear regression model and use this equation to calculate future concentrations.

1. Evaporate to dryness the supernatant collected during the cleaning of modified AuNPs (see step 7 in Subheading 3.3).

2. Resuspend the pellet in $1 \mathrm{~mL} \mathrm{TE}(1 \times)$.

3. Prepare a solution of Oligreen reagent $(2 \mu \mathrm{g} / \mathrm{mL})$ in TE $(1 \times)$.

4. Mix the resuspended supernatant with $1 \mathrm{~mL}$ Oligreen solution (see step 2 in Subheading 3.4.1). Incubate the solution for $5 \mathrm{~min}$ at room temperature protected from light.

5. Take $200 \mu \mathrm{L}$ of each solution prepared in step 4 and measure it in a plate reader (excitation $480 \mathrm{~nm}$, emission $520 \mathrm{~nm}$ ).

6. Interpolate the data obtained in the standard curve equation (see step 5 in Subheading 3.4.1) to determine the amount of unbounded oligonucleotide.

Oligonucleotides could be attached to AuNPs in a faster way using a dithiolane-based linker, which does not require a deprotection step, as in the case of thiols (Fig. 3).

1. Add the oligonucleotide to $1 \mathrm{~mL} 12 \mathrm{nM}$ of $13 \pm 2 \mathrm{~nm}$ gold nanoparticles (AuNP) (see Note 3).

2. Incubate the solution for $15 \mathrm{~min}$ at room temperature on a mini-shaker at a moderate speed.

3. Add $60 \mu \mathrm{L} \mathrm{NaCl}$ solution to a final concentration of $0.3 \mathrm{M}$ through the addition of small volumes (e.g., 5-10 $\mu \mathrm{L})($ see 


\subsubsection{AuNP}

Functionalization with Dithiolane-Modified Drugs
Note 5). Vortex the solution quickly after each addition of $\mathrm{NaCl}$ solution and incubate the sample for at least $10 \mathrm{~min}$ on a mini-shaker between each addition.

4. Incubate the sample for $4 \mathrm{~h}$ at room temperature on a minishaker at a moderate speed.

5. Continue with the washing steps, as described previously (see step 6 in Subheading 3.3) and the quantification of the unbound material (see Subheading 3.4) (see Notes 6 and 7).

In this case, the drugs have to be modified with a dithiolane-based linker (Fig. 4), and the AuNPs should be stabilized with oligonucleotides or PEG containing a sulfide-based linker. In this case, for $1 \mathrm{~mL}$ of $13 \pm 2 \mathrm{~nm} \mathrm{AuNP}(12 \mathrm{nM})$ add at least $2000 \mathrm{pmol}$ of stabilizing agent (e.g., PEG, oligonucleotide) and then the required amount of the modified drug for a total of $10,000 \mathrm{pmol}$ (stabilizing agent + drug) in the solution.

1. Add the stabilizing agent and incubate it for $15 \mathrm{~min}$ at room temperature on a mini-shaker at a moderate speed.

2. Add the modified drug very slowly and incubate it for $16 \mathrm{~h}$ at room temperature on a mini-shaker at a moderate speed (see Note 5 ).

3. Wash the nanoparticles as described in step 6 in Subheading 3.3 ( see Note 6).

4. Evaporate the collected supernatant to the initial volume ( $1 \mathrm{~mL}$ ) to quantify the drug and determine the nanoparticles' loading.

5. Measure the supernatant in a spectrophotometer (according to the specific absorbance of the drug) and calculate the unbound drug using the Beer-Lambert formula (see Note 8 ).

\section{Notes}

1. AuNP solution should be kept in darkness.

2. Keep TCEP under an inert atmosphere to prevent its oxidation. Once opened, store the compound in $20 \mu \mathrm{L}$ aliquots at $-20{ }^{\circ} \mathrm{C}$.

3. Duplexes should be annealed from their corresponding oligonucleotides before conjugation. In short, combine equal concentration and volume of each strand and add the same volume of annealing buffer $(3 \times)$. Then, incubate the sample at $95^{\circ} \mathrm{C}$ for $10 \mathrm{~min}$ and leave to cool slowly to room temperature. 


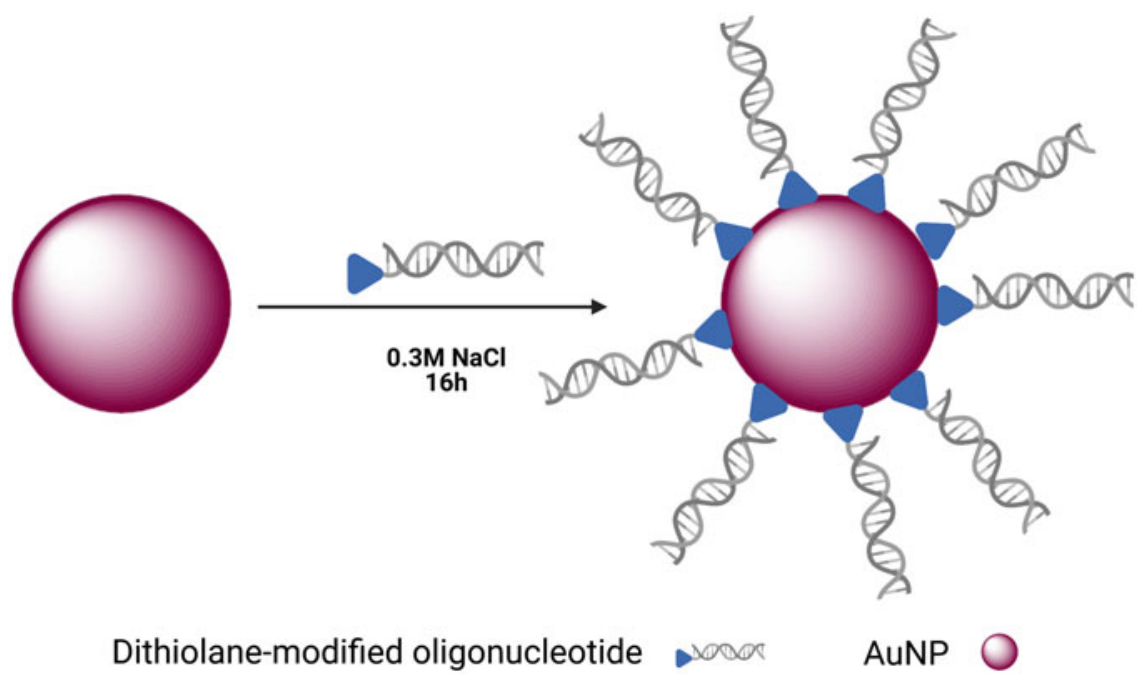

Fig. 3 Schematic representation of AuNPs functionalization with dithiolane-modified oligonucleotides

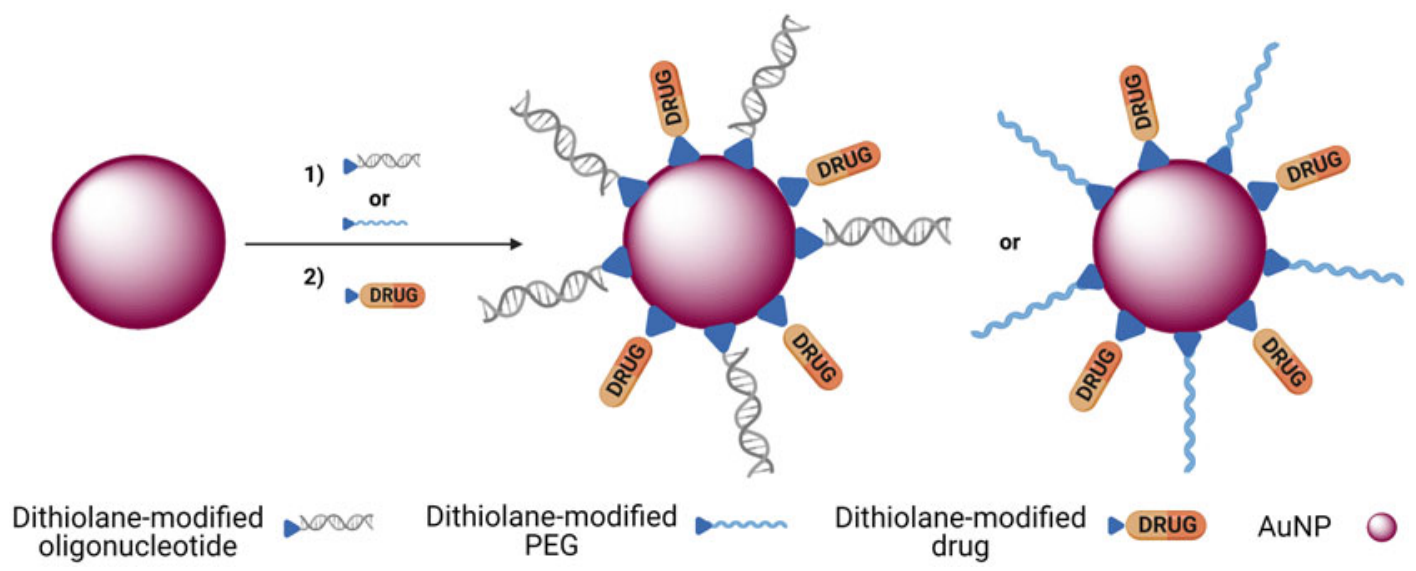

Fig. 4 Schematic representation of AuNPs functionalization with a dithiolane-modified drug using oligonucleotides or PEG as stabilizers

4. To get a complete functionalization of the nanoparticles, we recommend adding $10,000 \mathrm{pmol}$ of the oligonucleotide to $1 \mathrm{~mL}$ AuNP (12 nM, $13 \mathrm{~nm}$ ).

5. If you see that the AuNPs are being attached to the plastic tubes, move the solution to other plastic tubes immediately.

6. When AuNPs are changing their color to blue, it is due to aggregation. If vortexing the solution does not re-solubilize them, discard the preparation.

7. Oligonucleotide quantification could also be done by releasing the attached oligonucleotide [27]. To do so, treat the sample with $1 \mathrm{mM} \mathrm{GSH}$ for $8 \mathrm{~h} 37^{\circ} \mathrm{C}$. Then centrifuge the sample for 
$30 \mathrm{~min}$ at $13.2 \mathrm{rpm}$. Collect the supernatant and measure it as described in Subheading 3.3 and 3.4.

8. Drug quantification could also be done by comparing the drug supernatant absorbance or fluorescence with a proper standard linear calibration curve of the drug [19] or using analytical chromatography (HPLC).

\section{References}

1. Milán Rois P, Latorre A, Rodriguez Diaz C et al (2018) Reprogramming cells for synergistic combination therapy with nanotherapeutics against uveal melanoma. Biomimetics 3:28. https://doi org/10.3390/ biomimetics3040028

2. Hammond SM, Aartsma-Rus A, Alves S et al (2021) Delivery of oligonucleotide-based therapeutics: challenges and opportunities. EMBO Mol Med 13:el3243. https://doi.org/10. $15252 / \mathrm{emmm} .202013243$

3. Godfrey C, Desviat LR, Smedsrød B et al (2017) Delivery is key: lessons learnt from developing splice-switching antisense therapies. EMBO Mol Med 9:545-557. https:// doi.org/10.15252/emmm.201607199

4. Bregoli L, Movia D, Gavigan-Imedio JD et al (2016) Nanomedicine applied to translational oncology: a future perspective on cancer treatment. Nanomed Nanotechnol Biol Med 12: 81-103. https://doi.org/10.1016/j.nano. 2015.08.006

5. Gou Y, Miao D, Zhou M et al (2018) Bio-inspired protein-based nanoformulations for cancer theranostics. Front Pharmacol 9: 421-462. https://doi.org/10.3389/fphar. 2018.00421

6. Mahajan S, Patharkar A, Kuche K et al (2018) Functionalized carbon nanotubes as emerging delivery system for the treatment of cancer. Int J Pharm 548:540-558. https://doi.org/10. 1016/j.ijpharm.2018.07.027

7. Singh P, Pandit S, Mokkapati VRSS et al (2018) Gold nanoparticles in diagnostics and therapeutics for human cancer. Int J Mol Sci 19:1979. https://doi.org/10.3390/ ijms19071979

8. Mishra DK, Shandilya R, Mishra PK (2018) Lipid based nanocarriers: a translational perspective. Nanomed Nanotechnol Biol Med 14:2023-2050. https://doi.org/10.1016/j. nano.2018.05.021

9. Núñez C, Estévez SV, del Pilar CM (2018) Inorganic nanoparticles in diagnosis and treatment of breast cancer. J Biol Inorg Chem 23:
331-345. https://doi.org/10.1007/s00775018-1542-z

10. Manzano M, Vallet-Regí M (2018) Mesoporous silica nanoparticles in nanomedicine applications. J Mater Sci Mater Med 29:65. https:// doi.org/10.1007/s10856-018-6069-x

11. Pattni BS, Chupin VV, Torchilin VP (2015) New developments in liposomal drug delivery. Chem Rev 115:10938-10966. https://doi. org/10.1021/acs.chemrev.5b00046

12. Steinmetz NF (2013) Viral nanoparticles in drug delivery and imaging. Mol Pharm 10: 1-2. https://doi.org/10.1021/mp300658j

13. Daraee H, Eatemadi A, Abbasi E et al (2016) Application of gold nanoparticles in biomedical and drug delivery. Artif Cells Nanomed Biotechnol 44:410-422. https://doi.org/10. 3109/21691401.2014.955107

14. Turkevich J, Stevenson PC, Hillier J (1951) A study of the nucleation and growth processes in the synthesis of colloidal gold. Discuss Faraday Soc 11:55. https://doi.org/10.1039/ df9511100055

15. Cutler JI, Auyeung E, Mirkin CA (2012) Spherical nucleic acids. J Am Chem Soc 134: 1376-1391. https://doi.org/10.1021/ ja20935lu

16. Mokhtarzadeh A, Vahidnezhad H, Youssefian L et al (2019) Applications of spherical nucleic acid nanoparticles as delivery systems. Trends Mol Med 25(12):1066-1079. https://doi. org/10.1016/j.molmed.2019.08.012

17. Mioc A, Mioc M, Ghiulai R et al (2019) Gold nanoparticles as targeted delivery systems and theranostic agents in cancer therapy. Curr Med Chem 26(35):6493-6513. https://doi.org/ $10.2174 / 0929867326666190506123721$

18. Heo DN, Yang DH, Moon H-J et al (2012) Gold nanoparticles surface-functionalized with paclitaxel drug and biotin receptor as theranostic agents for cancer therapy. Biomaterials 33: 856-866. https://doi.org/10.1016/j. biomaterials.2011.09.064

19. Latorre A, Posch C, Garcimartín Y et al (2014) DNA and aptamer stabilized gold nanoparticles for targeted delivery of anticancer 
therapeutics. Nanoscale 6:7436-7442. https://doi.org/10.1039/C4NR00019F

20. Kapadia CH, Melamed JR, Day ES (2018) Spherical nucleic acid nanoparticles: therapeutic potential. BioDrugs 32:297-309. https:// doi.org/10.1007/s40259-018-0290-5

21. Randeria PS, Seeger MA, Wang X-Q et al (2015) siRNA-based spherical nucleic acids reverse impaired wound healing in diabetic mice by ganglioside GM3 synthase knockdown. Proc Natl Acad Sci 112:5573-5578. https://doi.org/10.1073/pnas.1505951112

22. Wang X, Hao L, Bu HF et al (2016) Spherical nucleic acid targeting microRNA-99b enhances intestinal MFG-E8 gene expression and restores enterocyte migration in lipopolysaccharide-induced septic mice. Sci Rep 6:1-13. https://doi.org/10.1038/ srep31687

23. Latorre A, Posch C, Garcimartín Y et al (2014) Single-point mutation detection in RNA extracts using gold nanoparticles modified with hydrophobic molecular beacon-like structures. Chem Commun 50(23):3018-3020. https://doi.org/10.1039/c3cc47862a

24. Coutinho C, Somoza Á (2019) MicroRNA sensors based on gold nanoparticles. Anal Bioanal Chem 411(9):1807-1824

25. Posch C, Latorre A, Crosby MB et al (2015) Detection of GNAQ mutations and reduction of cell viability in uveal melanoma cells with functionalized gold nanoparticles. Biomed Microdev 17:15-37. https://doi.org/10. 1007/s10544-014-9908-7

26. Liu X, Atwater M, Wang J, Huo Q (2007) Extinction coefficient of gold nanoparticles with different sizes and different capping ligands. Colloids Surf B Biointerfaces 58:3-7. https://doi.org/10.1016/J.COLSURFB. 2006.08.005

27. Melamed JR, Riley RS, Valcourt DM et al (2017) Quantification of siRNA duplexes bound to gold nanoparticle surfaces. Methods Mol Biol 1570:1-15

Open Access This chapter is licensed under the terms of the Creative Commons Attribution 4.0 International License (http://creativecommons.org/licenses/by/4.0/), which permits use, sharing, adaptation, distribution and reproduction in any medium or format, as long as you give appropriate credit to the original author(s) and the source, provide a link to the Creative Commons license and indicate if changes were made.

The images or other third party material in this chapter are included in the chapter's Creative Commons license, unless indicated otherwise in a credit line to the material. If material is not included in the chapter's Creative Commons license and your intended use is not permitted by statutory regulation or exceeds the permitted use, you will need to obtain permission directly from the copyright holder. 